\title{
Evaluación de la capacidad pulmonar en función del volumen corriente en estudiantes universitarios durante la pandemia por la COVID-19
}

\section{Evaluation of lung capacity as a function of current volume in university students during the COVID-19 pandemic}

\author{
Solange Marcelina Espinoza Quispe 1,a, Lim Liu Injante, Lim 1,a, Junior Ernesto Melo Torres 1,a,

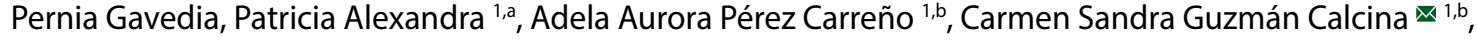 \\ Yuliana Ayala Piñella ${ }^{1, b}$, Jessenia Brillit Villaverde Herrera ${ }^{2, b}$
}

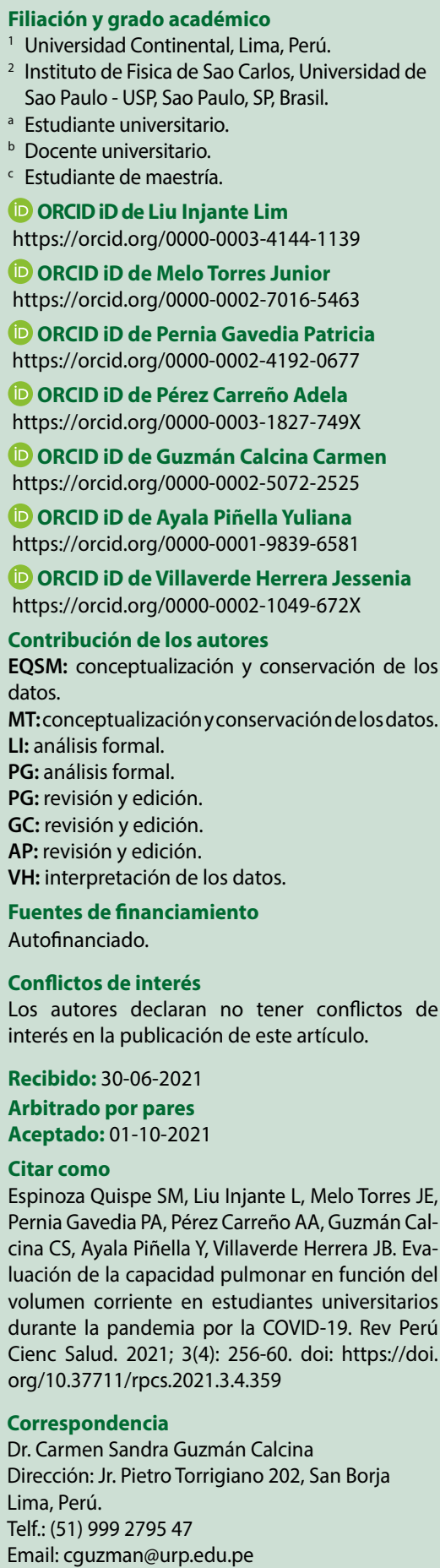

\section{RESUMEN}

Objetivo. Evaluar la capacidad pulmonar en función del volumen corriente (VC) y analizar su influencia en presencia de diversos factores externos, tales como actividad física (natación), alimentación e infección respiratoria. Métodos. El estudio fue de tipo observacional, descriptivo, transversal y prospectivo. Se realizó una encuesta online a una muestra aleatoria conformada por 50 estudiantes universitarios entre 17 y 20 años. Se midió el VC mediante el uso de un espirómetro casero y se clasificó bajo el programa IBM SPSS Statistics-24, para los respectivos análisis estadísticos. Resultados. De las encuestas, se obtuvieron los siguientes datos: el VC promedio de los estudiantes que practican natación fue de $(797,0 \pm 2,0) \mathrm{ml}$. El VC promedio de los alumnos que siguen una alimentación saludable fue de $(567,00 \pm$ $2,0) \mathrm{ml}$ y el VC promedio de aquellos con antecedentes respiratorios fue de $(429,0 \pm 2,0) \mathrm{ml}$. Conclusiones. Tener una dieta rica en frutas y verduras o realizar alguna actividad física aumenta en gran medida la capacidad pulmonar del individuo.

Palabras clave: espirometría; capacidad pulmonar; volumen corriente; volumen de reserva inspiratoria; volumen de reserva espiratoria; capacidad vital; capacidad vital forzada (Fuente: DeCS BIREME).

\begin{abstract}
Objective. To evaluate lung capacity as a function of current volume (CV) and to analyze its influence in the presence of various external factors, such as physical activity (swimming), feeding and respiratory infection. Methods. The study was observational, descriptive, transversal and prospective. An online survey was conducted on a random sample of 50 university students between the ages of 17 and 20. CV was measured using a home spirometer and was classified under the IBM SPSS Statistics-24 program for statistical analysis. Results. From the surveys, the following data was obtained: the average CV of swimming students was $(797.0 \pm 2.0) \mathrm{ml}$. The average CV of students following a healthy diet was $(567.00 \pm 2.0) \mathrm{ml}$ and the average CV of those with a respiratory history was $(429.0 \pm 2.0) \mathrm{ml}$. Conclusions. Having a diet rich in fruits and vegetables or having some physical activity greatly increases the lung capacity of the individual.
\end{abstract}

Keywords: spirometry, lung capacity, current volume, inspiratory reserve, expiratory reserve, vital capacity, forced vital capacity (Source: MeSH NLM). 


\section{INTRODUCCIÓN}

Desde los inicios del año 2020, la población mundial se ha visto afectada debido a la grave crisis sanitaria y al aislamiento social, como medidas urgentes ante la propagación de la COVID-19; por consecuencia, la grave preocupación por el riesgo de transmisión del virus SARSCoV-2 en el contexto de la realización de pruebas de función pulmonar ha sido un tema de reciente estudio. Una de las pruebas de función respiratoria (PFR) más básicas es la espirometría.

La espirometría es un tipo de prueba de función pulmonar que permite la medición del volumen corriente (VC) y el flujo de aire que entra y sale de los pulmones durante el proceso ventilatorio de inspiración y espiración. La presente técnica permite, además de evaluar la capacidad de los pulmones para oxigenar eficientemente la sangre, diagnosticar la presencia de potenciales enfermedades respiratorias, así como diferenciar entre anomalías respiratorias y su probable origen, pulmonar, cardíaco, neurológico u otro ${ }^{(1)}$.

Según el Foro de las Sociedades Respiratorias Internacionales, la sobrecarga sanitaria de enfermedades respiratorias a nivel mundial va en aumento. La exposición a agresivos ambientes externos debido a la intensa contaminación ambiental, el excesivo consumo de tabacos o la mala nutrición, entre otros factores, conlleva a graves y silenciosas consecuencias que podrían afectar a la salud. La evaluación pulmonar es fundamental para tratar tempranas enfermedades respiratorias de manera eficiente; por ello, es necesario analizar sus variaciones respectivas en diversas condiciones y muestras de estudio ${ }^{(2,3)}$

En su investigación, Padilla ${ }^{(4)}$ estudió a un grupo de 32 atletas en una probable disparidad físico-deportiva entre resistencias central y periférica mediante espirometría. Con la marca o tiempo de su mejor desempeño se obtuvo, en promedio, que el grupo de karate fue el menos favorecido por los indicadores de adaptación en la resistencia físicodeportiva músculo esquelético y central en comparación con los grupos de natación, medio fondo y de resistencia; concluyéndose que solamente entre las nadadoras fue significativa la proporción inversa entre pulso máximo de oxígeno y la frecuencia cardiaca máxima ${ }^{(4)}$.

En México, Regalado et al. ${ }^{(5)}$ evaluaron el efecto del tabaquismo, los síntomas respiratorios y el asma sobre la función pulmonar espirométrica en una población adulta. Mediante un muestreo a 919 personas entre los 14 y 86 años, se realizaron pruebas espirométricas y encuestas estandarizadas. Los resultados mostraron que el asma y los síntomas respiratorios disminuyeron la función pulmonar en hombres, así como el tabaquismo en mujeres, específicamente en el volumen espiratorio forzado (VEF) ${ }^{(5)}$.

Capió et al. ${ }^{(6)}$, analizaron estudios de la función pulmonar en personas obesas con obesidad grado I, II, III o mórbida. Mediante diversas pruebas de función respiratoria realizadas, incluyendo la prueba de espirometría lenta y forzada, observaron que el índice de masa corporal (IMC), estaba asociado proporcionalmente con el cociente entre el VEF y la capacidad vital forzada (FVC), asociado a una reducción del VC en todos los grupos medidos; por tanto, concluyeron que los diversos tipos de obesidad causados por una mala alimentación tienen implicaciones negativas en la función pulmonar $y$, por ende, en la salud ${ }^{(6)}$.

Los espirómetros actuales se basan en las técnicas de la computación electrónica y son ampliamente utilizados como medio de diagnóstico en la mayoría de los hospitales modernos. Las tecnologías modernas hacen posible el uso de este equipamiento en consultas de clínicas privadas, fuera de hospitales, así como en las oficinas personales de especialistas en función pulmonar ${ }^{(7,8)}$.

\section{En la espirometría simple se obtienen:}

- Volumen corriente (VC): es la cantidad de aire que se utiliza en cada respiración (inspiración y espiración) no forzada, es decir, el aire utilizado durante el ciclo respiratorio. Por convenio se mide el volumen espirado ya que normalmente el inspirado y el espirado no son idénticos. Es aproximadamente de $500 \mathrm{ml}$.

- Volumen de reserva inspiratoria (VRI): es la cantidad máxima de volumen de aire que se puede inspirar partiendo del VC. Es de aproximadamente $3000 \mathrm{ml}$.

- Volumen de reserva espiratoria (VRE): es la cantidad máxima de volumen de aire que se puede espirar partiendo del VC y bajo éste. Es aproximadamente de $1100 \mathrm{ml}$.

- Capacidad vital (CV): es el volumen máximo que somos capaces de inspirar y espirar en condiciones normales, y resulta de la suma del VC y los VRI y VRE.

- La capacidad vital forzada (CVF) es la capacidad máxima de captar y expulsar aire en condiciones forzadas, por lo que en condiciones normales será mayor la CVF que la CV; no obstante, en enfermedades con patrón obstructivo esto se invierte.

- Otro volumen importante que no se puede medir con el espirómetro es el volumen residual (VR), el cual es el volumen de aire que queda en los 


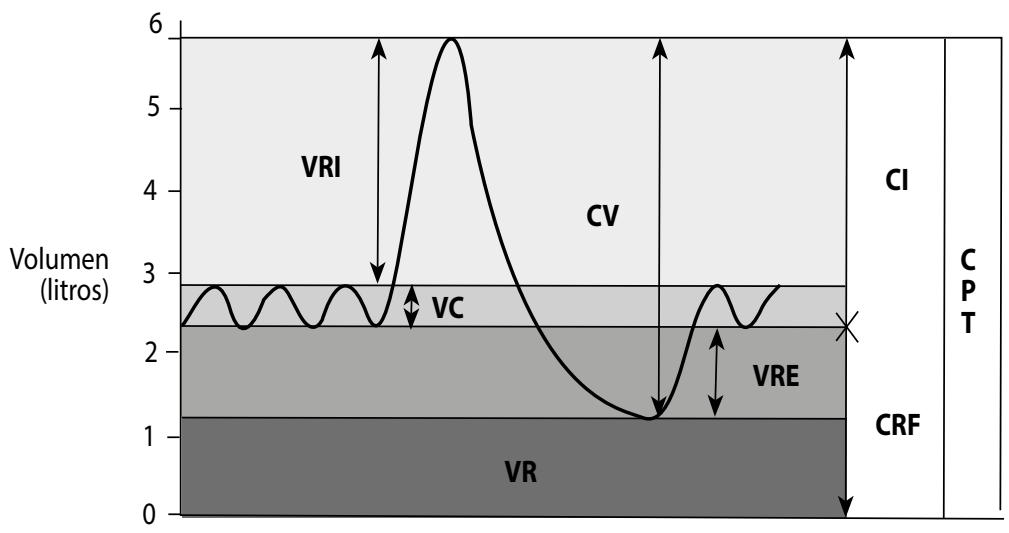

Figura 1. Volúmenes y capacidades pulmonares estáticos ${ }^{(10)}$

pulmones al final de una espiración máxima sin poder ser liberado de los pulmones. Este volumen solo se pierde cuando cesa la función pulmonar, es decir, el óbito. ElVR es de aproximadamente $1200 \mathrm{ml}$.

- Sumando la CV con el VR se obtiene la capacidad pulmonar total (CPT) ${ }^{(9)}$.

El presente trabajo tiene como objetivo principal evaluar la capacidad pulmonar de los estudiantes universitarios mediante la obtención del VC usando un espirómetro casero de agua y, en consecuencia, analizar su influencia en presencia de diversos factores externos como actividad física (natación), alimentación e infección respiratoria

\section{MÉTODOS}

\section{Tipo de estudio}

La investigación fue de enfoque cunatitativo, de tipo observacional, descriptiva, transversal, prospectiva y predictiva.

\section{Población y muestra}

La muestra aleatoria, realizada en noviembre del 2020, está constituida por 50 jóvenes universitarios, entre hombres y mujeres en el rango de 17 y 20 años de edad, matriculados en la Facultad de Medicina Humana de una universidad en el departamento de Lima.

\section{Instrumentos de recolección de datos}

Como instrumento de medición se elaboró un espirómetro de agua construido como parte del trabajo y diseñado bajo las recomendaciones y estándares de la Sociedad Americana del Tórax (ATS), Asociación Americana de Cuidado Respiratorio (AARC), Comité Respiratorio Europeo (ERC), Administración de Salud y Seguridad Ocupacional
(OSHA) e Instituto Nacional para la Salud y Seguridad Ocupacional (NIOSH) (11). Para la validez del instrumento se ha considerado la evidencia relacionada con el contenido, ya que fueron tomados en cuenta todos los factores que pueden influir en los resultados. Así mismo, se realizó una encuesta online en la plataforma de Google Forms, para registrar toda la información. Se emplearon los siguientes materiales: botella con capacidad de 7 litros, una manguera de tres metros, una batea y agua.

\section{Procedimientos de la recolección de datos}

Para llevar a cabo la técnica de recolección de datos, se les pidió a los estudiantes que inhalen aire y luego soplen por una manguera para luego conocer su capacidad pulmonar. Posteriormente, se comparó los datos con los datos investigados en los artículos. Se obtuvieron tres medidas en cada caso.

\section{Análisis de datos}

Estos datos se compararon con los resultados de la prueba espirométrica y de ahí surgieron los criterios de normalidad para interpretar los resultados y emitir el diagnóstico ${ }^{(7,11)}$. Finalmente, se contrastaron los resultados obtenidos con los resultados de otros autores. Para realizar el diagnóstico espirométrico es necesario disponer de valores de referencia, conocidos como valores predichos o normales, que se obtienen de evaluar fórmulas de regresión desarrolladas a partir de muestras de la población en estudio.

Para el análisis descriptivo e inferencial de datos se utilizó el programa IBM SPSS Statistics-24. Se obtuvieron los estadísticos descriptivos de las variables influyentes VC1, VC2 y VC3 para los diferentes análisis de la muestra, obteniendo la media y desviación estándar de dichas variables cuantitativas. 
Tabla 1. Datos de las encuestas a los 50 estudiantes de la comunidad universitaria

\begin{tabular}{lcccc}
\hline & \multicolumn{2}{c}{$\begin{array}{c}\text { Mujer entre } \\
\mathbf{1 7 - 2 0} \text { años }\end{array}$} & \multicolumn{2}{c}{$\begin{array}{c}\text { Varón entre 17- } \\
\text { 20 años }\end{array}$} \\
\cline { 2 - 5 } & Sí & No & Sí & No \\
\hline Infección respiratoria & 9 & 16 & 10 & 15 \\
Asma & 6 & 19 & 11 & 14 \\
Uso de aerosoles & 3 & 22 & 8 & 17 \\
Uso de broncodilatadores & 2 & 23 & 9 & 16 \\
Olvido de uso de los & 12 & 13 & 13 & 12 \\
broncodilatadores & 0 & 25 & 6 & 19 \\
Operaciones en este año & 11 & 14 & 13 & 12 \\
Fumador pasivo & 15 & 10 & 18 & 7 \\
Actividad física & 5 & 20 & 14 & 11 \\
$\begin{array}{l}\text { Practica natación } \\
\text { Consumo saludable (frutas, }\end{array}$ & 22 & 3 & 20 & 5 \\
verduras, etc.) & & & & \\
\hline
\end{tabular}

\section{RESULTADOS}

Realizada la encuesta dentro de la muestra de los estudiantes universitarios, se obtuvieron las siguientes clasificaciones: el $66 \%$ realizaba actividad física (30\% femenino y $36 \%$ masculino), el $38 \%$ practicaba natación (10\% femenino y $28 \%$ masculino), el $84 \%$ tenía una alimentación balanceada ( $44 \%$ femenino y $40 \%$ masculino), y el $38 \%$ presentaba infecciones respiratorias (18\% femenino y $20 \%$ masculino). Adicionalmente a ello, el $34 \%$ presentaba antecedentes de asma ( $12 \%$ femenino y $22 \%$ masculino), el $48 \%$ eran fumadores ( $22 \%$ femenino y $26 \%$ masculino), el $22 \%$ usaban broncodilatadores ( $4 \%$ femenino y $18 \%$ masculino) (ver Tabla 1). Todos los datos fueron obtenidos durante la pandemia por la COVID-19.

En la gráfica 1 se calculó el VC promedio de los tres subgrupos mencionados. En el primer subgrupo (de nadadores) se obtuvo un valor de VC de 797,0 $\pm 2,0 \mathrm{ml}$. En el segundo subgrupo, conformado por participantes que siguen una dieta rica en verduras, se obtuvo un valor aproximado de 567,0 $\pm 2,0 \mathrm{ml}$. Finalmente, el VC promedio obtenido de las personas con antecedentes respiratorios (AR) fue de $429,0 \pm 2,0 \mathrm{ml}$.

\section{DISCUSIÓN}

El VC promedio de los nadadores en el presente estudio fue de 797,0 $\pm 2,0 \mathrm{ml}$, una cifra similar al estudio de Padilla ${ }^{(4)}$, cuyo valor aproximado fue de $834,7 \mathrm{ml}$ realizado a una muestra similar. Así mismo, el VC promedio en participantes que siguen una alimentación saludable

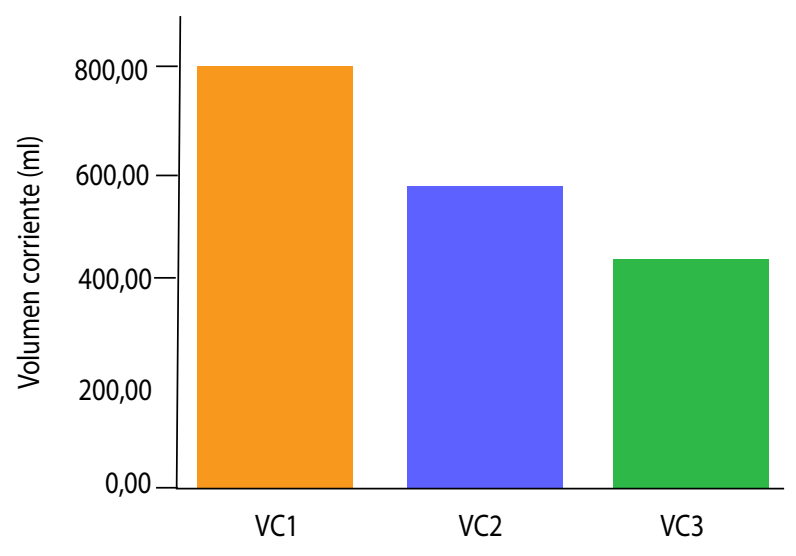

Figura 2. Volumen corriente en función a las variables influyentes VC1 (nadadores), VC2 (dieta rica en verduras) y VC3 (antecedentes respiratorios)

fue de $567,0 \pm 2,0 \mathrm{ml}$, resultado semejante al obtenido por Fernández ${ }^{(13)}$, en Murcia, donde fue de $567,79 \mathrm{ml}$. Finalmente, el VC promedio obtenido de las estudiantes con antecedentes respiratorios fue de $429,0 \pm 2,0 \mathrm{ml}$, cuyo valor aproximado se asemeja frente a $428,27 \mathrm{ml}$ en el estudio realizado por De los Santos ${ }^{(14)}$.

Los resultados demuestran que realizar moderadamente ejercicio físico logra el aumento de la capacidad pulmonar de la persona, ya que aumenta el tamaño y la elasticidad de los pulmones. Además, tener una dieta balanceada, como se sabe, aporta gran cantidad de fibras al tejido de los pulmones, dándole una mayor resistencia y elasticidad; en consecuencia, ello se ve reflejado en el aumento del VC $y$, por tanto, de la capacidad pulmonar. Así mismo, los estudios demuestran que el consumo de diversas sustancias tóxicas perjudica directamente la capacidad pulmonar, específicamente en personas con alta incidencia en problemas respiratorios.

Del presente trabajo, se concluye por último que:

- La capacidad pulmonar en las personas que practican frecuentemente el deporte de la natación logran desarrollar su capacidad pulmonar en su totalidad gracias a su aporte en la alta resistencia del sistema respiratorio. Además de ello, reduce el riesgo de padecer futuras enfermedades cardiacas.

- La capacidad pulmonar presenta una relación directamente proporcional al estilo de vida saludable, lo que comprende el ejercicio físico y una dieta saludable. Es decir, el incremento de la fibra en la dieta (frutas y verduras, el consumo de alimentos con propiedades antioxidantes $y$ antiinflamatorias, entre otras recomendaciones), fortalecerá las propiedades musculares de los pulmones. 
- Por último, el constante consumo de sustancias tóxicas (humo de cigarrillos, etc.) conlleva a muchas consecuencias no deseadas, como enfermedades cardiacas, pulmonares, etc.; $y$, por ende, a una disminución en la capacidad pulmonar debida a la dificultad para respirar.

\section{REFERENCIAS}

1. American Thoracic Society (ATS). Standardization of Spirometry, 1994 Update. Am J of Respir Crit. Care Med [Internet]. 1995 Sep [Consultado 2020 Nov 15]; 152(3): 1107-36. DOI:10.1164/ajrccm.152.3.7663792.

2. Foro de las Sociedad Respiratorias Internacionales. El impacto mundial de la Enfermedad Respiratoria [Internet] 2017 [Consultado 2020 Nov 15]. Disponible en: https://www.who.int/gard/publications/The_Global_Impact_of_Respiratory_Disease_ES.pdf

3. Nutrición y salud pulmonar en enfermedades crónicas respiratorias [Internet]. Madrid: Fundación LovexAir; 2020 [Consultado 2020 Nov 22]. Disponible en: https:// www.lovexair.com/news/noticias-generales/7/7-Nutricion-y-salud-pulmonar-en-enfermedades-respiratorias-cronicas\#: :text=Las\%20infecciones\%20respiratorias\%20como\%20las,el\%20desarrollo\%20de\%20 todas\%20ellas

4. Padilla PJ. Estudio. Comparativo de la fisiología del ejercicio en grupos de deportistas mexicanas de diferentes especialidades [Internet]. Instituto Nacional de Enfermedades Respiratorias; 2001 [Consultado 2020 Nov 18]; 14 (1): 145-150. Disponible en: https://www.medigraphic.com/pdfs/iner/in-2001/in013b.pdf

5. Regalado PJ, Gómez A, Ramírez J, Vázquez JC. Efectos del tabaquismo, los síntomas respiratorios y el asma sobre la espirometría de adultos de la ciudad de México. Instituto Nacional de Enfermedades Respiratorias. 2018; 47(5): 327-334.
6. Capió C, Santiago A, García A, Álvarez R. Función pulmonar y obesidad [Internet]. 2014 [Consultado 2020 Nov 18]; 30(5): 1054-1062. DOI: https://dx.doi.org/10.3305/ nh.2014.30.5.8042

7. Almirall J. y Begin P. Interpretación automatizada de la Espirometría. Quebec: Unidad de Investigación Clínica, Complejo Hospitalario de la Sagamie, Chicoutimi; 1998.

8. Gold WM, Koth L. Pulmonary Function Equipment, Spirometers. J Medical Electronics [Internet]. 2016 [Consultado 2020 Oct 12]; 168: 84-85. DOI: 10.1016/B978-14557-3383-5.00025-7

9. Grupo MBE. Galicia integrada en la Red Temática de Investigación sobre Medicina Basada en la Evidencia Técnicas en AP Espirometría forzada [Internet] [Consultado 2020 Mar]. Disponible en: http://www.fisterra.com/material/tecnicas/espirometria/espirometria.pdf

10. Espirometría [Internet] Wikipedia [Consultado 2020 Oct 12]. Disponible en: https://es.wikipedia.org/wiki/Espirometr\%C3\%ADa

11. Beltrán R. Diseño e implementación de un espirómetro. Revista Tekhnê. 2013; 10(2): 5-14.

12. American Thoracic Society (ATS). Lung Function Testing: Selection of reference values and interpretative strategies. Medical Section of the American Lung Association [Internet]. 1991 [Consultado 2020 Nov 10]; 144: 12021218. DOI: $10.1164 /$ ajrccm/144.5.1202

13. Fernández $A$, Torres $M L$, Mosteiro $M$, Represas $C$, Botana MI, Nuñez $M$, et al. Utilización de la espirometría en los centros de atención primaria de Galicia. Pneuma [Internet]. 2005 [Consultado 2020 Oct 28]; 1(2): 80-84. Disponible en: https://www.sogapar.info/wp-content/ uploads/2010/08/pneuma-n-2-3.pdf

14. De los Santos JM, Peña C, Ortega R, Manso AB. Revisión de evidencias sobre la influencia de la dieta en la evolución de la EPOC. Enferm. Glob [Internet]. 2012. [Consultado 2020 Oct 28]; 11(28): 323-336. Disponible en: https://scielo.isciii.es/scielo.php?script=sci_arttext\&pi$d=$ S1695-61412012000400017 